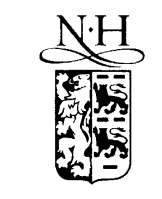

\title{
Classical theory for scattering of rigid molecules from surfaces
}

\author{
J. Dai, J.R. Manson * \\ Department of Physics and Astronomy, Clemson University, Clemson, SC 29634, USA
}

\begin{abstract}
A theoretical model is presented for the scattering of molecules from surfaces under conditions in which the major modes of energy transfer are multiple phonon excitation at the surface and rotational excitations of the molecule. Beginning from a completely quantum mechanical formalism, the classical limit is obtained for large mass molecules and large energies and in this limit the result is a closed-form expression for the scattering intensities. Results of calculations are compared with recent measurements for the scattering of hyperthermal beams of $\mathrm{C}_{2} \mathrm{H}_{2}$ from a $\operatorname{LiF}\left(\begin{array}{ll}0 & 0\end{array}\right)$ surface. The results of these comparisons indicate that a classical theoretical treatment of translational and rotational motion is adequate for describing this system. (c) 2002 Elsevier Science B.V. All rights reserved.
\end{abstract}

In this paper a theoretical model is developed for the scattering of rigid molecules from surfaces where the modes of energy exchange are multiphonon excitation of the surface and rotational excitation of the molecule and both are treated in the classical mechanical limit. Classical mechanics has been shown adequate to explain the scattering of heavier atoms from surfaces [1,2] where large numbers of phonon transfers is the means of energy exchange. Classical mechanics even gives good descriptions of the energy transfer for the surface collisions of small mass projectiles such as $\mathrm{He}$ and $\mathrm{D}_{2}$ at incident energies over $100 \mathrm{meV}$ [3]. For molecules with masses substantially larger than hydrogen or deuterium, such as $\mathrm{CO}, \mathrm{CO}_{2}$ or $\mathrm{C}_{2} \mathrm{H}_{2}$, rotational quantum numbers even at temperatures as low as $100 \mathrm{~K}$ are of order 10 or larger and in a typical surface collision the rotational

\footnotetext{
${ }^{*}$ Corresponding author. Fax: +1-864-656-0805.

E-mail address: jmanson@clemson.edu (J.R. Manson).
}

excitation can involve quantum numbers even larger than that. Thus for such molecules, a classical treatment of the rotational excitations, combined with a classical treatment of the phonon exchange should be adequate.

An earlier treatment of this same problem has been presented based on treating the rotational interactions of a molecular beam incident on the surface as interactions with a surface of discrete scattering centers [4]. This earlier treatment obtained very good agreement with recent measurements of the angular distributions of $\mathrm{C}_{2} \mathrm{H}_{2}$ [5] scattering from a $\operatorname{LiF}(001)$ surface at incident energies above $100 \mathrm{meV}$. Qualitative agreement was obtained with further experimental measurements of the scattered intensities as a function of final rotational energies. The work described here extends this earlier theoretical model to include a better model of the surface interaction, one in which the rotational interaction is with a surface having a smoothly varying repulsive barrier. In this case, the correct conservation of angular momentum perpendicular to the surface is included, 
as well as the correct conservation of linear momentum parallel to the surface.

The basic results of this theoretical treatment are presented in analytical closed-form equations, which makes the results useful for predicting the effect on the scattered distributions due to changes in the incident conditions. Calculations are carried out and compared with the $\mathrm{C}_{2} \mathrm{H}_{2}$ experimental measurements. The present model predicts the measured total angular distribution lobes equally well or better than the previous work [4]. Furthermore, the present results for the intensities as functions of final rotational energy and for the final observed rotational temperatures as functions of the incident beam translational energy are significantly better. Although this work seems to indicate that, in order to obtain completely quantitative agreement with experiment, it will be necessary to include the possibility of excitation of internal molecular vibrational modes, it clearly points out the importance of imposing the proper conditions of momentum and angular momentum conservation at the surface.

The surface scattering of a molecule, including both translational and rotational motion, can be developed from several different starting points, but a useful approach is that of Brako and Newns [6] for scattering of atomic projectiles from a smooth surface. In this case, there is no rotational motion and the probability for a projectile to exchange translational energy $E_{\mathrm{f}}^{\mathrm{T}}-E_{\mathrm{i}}^{\mathrm{T}}$ and momentum parallel to the surface $\boldsymbol{P}=\boldsymbol{P}_{\mathrm{f}}-\boldsymbol{P}_{\mathrm{i}}$, in making a transition from an initial state defined by momentum $\boldsymbol{p}_{\mathrm{i}}$ to final state $\boldsymbol{p}_{\mathrm{f}}$, is given by

$$
\begin{aligned}
N\left(\boldsymbol{p}_{\mathrm{f}}, \boldsymbol{p}_{\mathrm{i}}\right)=\langle & \langle| S^{\dagger} \delta\left(\widehat{H}-\varepsilon_{\mathrm{i}}+E_{\mathrm{f}}^{\mathrm{T}}-E_{\mathrm{i}}^{\mathrm{T}}\right) \\
& \left.\times \delta\left(\widehat{\boldsymbol{P}}-\boldsymbol{K}_{\mathrm{i}}+\boldsymbol{P}_{\mathrm{f}}-\boldsymbol{P}_{\mathrm{i}}\right) S|\rangle\right\rangle,
\end{aligned}
$$

where $\widehat{H}$ is the total Hamiltonian, $\varepsilon_{\mathrm{i}}$ is the initial energy of the target surface, $\boldsymbol{K}_{\mathrm{i}}$ is the initial parallel momentum of the target, $\widehat{\boldsymbol{P}}$ is the parallel momentum operator for the target and the double brackets $\langle\langle\rangle\rangle$ define an ensemble average over all initial target states. $S$ is the scattering operator defined by

$S=\lim _{t \rightarrow \infty} U(+t,-t)$,

where $U(+t,-t)$ is the time-evolution operator.
The differential reflection coefficient giving the fraction of the incident beam scattered into a small final solid angle $\mathrm{d} \Omega_{\mathrm{f}}$ and small range of final translational energy $\mathrm{d} E_{\mathrm{f}}^{\mathrm{T}}$, the quantity usually measured in an experiment, is related to Eq. (1) by a simple density of states

$\frac{\mathrm{d} R\left(\boldsymbol{p}_{\mathrm{f}}, \boldsymbol{p}_{\mathrm{i}}\right)}{\mathrm{d} E_{\mathrm{f}}^{\mathrm{T}} \mathrm{d} \Omega_{\mathrm{f}}}=p_{\mathrm{f}} p_{f z} N\left(\boldsymbol{p}_{\mathrm{f}}, \boldsymbol{p}_{\mathrm{i}}\right)$.

When this formalism is extended to the classical limit of atomic scattering, where the de Broglie wavelengths of the projectile are short, the resulting differential reflection coefficient, explained in fuller detail below, is given by

$$
\begin{aligned}
\frac{\mathrm{d} R\left(\boldsymbol{p}_{\mathrm{f}}, \boldsymbol{p}_{\mathrm{i}}\right)}{\mathrm{d} E_{\mathrm{f}}^{\mathrm{T}} \mathrm{d} \Omega_{\mathrm{f}}}= & \frac{m^{2} p_{\mathrm{f}} v_{\mathrm{R}}^{2} L^{4}}{8 \pi^{2} \hbar^{2} p_{i z} S_{\mathrm{uc}}}\left|\tau_{f i}\right|^{2}\left(\frac{\pi}{k_{\mathrm{B}} T_{S} \Delta E_{0}}\right)^{3 / 2} \\
& \times \exp \left\{-\frac{\left(E_{\mathrm{f}}-E_{\mathrm{i}}+\Delta E_{0}\right)^{2}+2 v_{\mathrm{R}}^{2} P^{2}}{4 k_{\mathrm{B}} T_{S} \Delta E_{0}}\right\},
\end{aligned}
$$

where $\Delta E_{0}=\boldsymbol{p}^{2} / 2 M_{C}$ with $\boldsymbol{p}=\boldsymbol{p}_{\mathrm{f}}-\boldsymbol{p}_{\mathrm{i}}$ is the translational recoil energy, $T_{S}$ is the temperature, $m$ is the projectile mass, $S_{\mathrm{uc}}$ is the area of a surface unit cell, $L$ is a quantization length and $v_{\mathrm{R}}$ is a weighted average over phonon velocities parallel to the surface [6]. The effects of the interaction potential are contained in the scattering form factor $\left|\tau_{f i}\right|^{2}$ which is the square modulus of the ofenergy-shell transition matrix of the elastic part of the interaction potential [9]. Eq. (4) describes scattering in the classical limit of a particle colliding with a surface that is smooth except for vibrational motion. Since it is classical, it results from large numbers of multiphonon transfers, but correct conservation of parallel momentum with each phonon transfer is insured by the momentum $\delta$-function in Eq. (1) above. It is this parallel momentum conservation that produces the Gaussianlike function in $\boldsymbol{P}$.

Now considering the extension to molecular scattering, rotational motion must be accounted for in addition to translational motion. At a smooth surface, angular momentum of the rotating molecule is not conserved in the directions parallel to the surface because of the broken symmetry caused by the presence of the surface. 
However, in the direction perpendicular to the surface, the angular momentum of surface and projectile is conserved in all rotational energy exchanges. Thus the extension to Eq. (1) above for describing a molecule making a transition from a given initial state described by momentum $\boldsymbol{p}_{\mathrm{i}}$ and angular momentum $\boldsymbol{l}_{\mathrm{i}}$ to final state $\left\{\boldsymbol{p}_{\mathrm{f}}, \boldsymbol{l}_{\mathrm{f}}\right\}$ is

$$
\begin{aligned}
N\left(\boldsymbol{p}_{\mathrm{f}}, \boldsymbol{l}_{\mathrm{f}} ; \boldsymbol{p}_{\mathrm{i}}, \boldsymbol{l}_{\mathrm{i}}\right)= & \left\langle\langle | S ^ { \dagger } \delta \left(\widehat{H}-\varepsilon_{\mathrm{i}}+E_{\mathrm{f}}^{\mathrm{T}}-E_{\mathrm{i}}^{\mathrm{T}}\right.\right. \\
& \left.+E_{\mathrm{f}}^{\mathrm{R}}-E_{\mathrm{i}}^{\mathrm{R}}\right) \delta\left(\widehat{\boldsymbol{P}}-\boldsymbol{K}_{\mathrm{i}}+\boldsymbol{P}_{\mathrm{f}}-\boldsymbol{P}_{\mathrm{i}}\right) \\
& \left.\times \delta\left(\hat{l}_{z}-\mathscr{L}_{z i}+l_{f z}-l_{i z}\right) S|\rangle\right\rangle,
\end{aligned}
$$

where $E_{\mathrm{f}}^{\mathrm{R}}-E_{\mathrm{i}}^{\mathrm{R}}$ is the rotational energy transferred between the final and initial state of the scattered molecule, $\hat{l}_{z}$ is the momentum operator for the direction $z$ perpendicular to the surface, $\mathscr{L}_{z i}$ refers to the initial momentum state of the surface and again the double brackets signify an average over all initial states of the surface.

Upon Fourier transforming the momentum and energy $\delta$-functions in Eq. (5) and taking the semiclassical limit, the differential reflection coefficient can be expressed in terms of a Fourier transform of time dependent scattering kernels, $K_{\mathrm{T}}\left(t, T_{S}\right)$ for translational motion and $K_{\mathrm{R}}\left(t, T_{S}\right)$ for rotational motion

$$
\begin{aligned}
N\left(\boldsymbol{p}_{\mathrm{f}}, \boldsymbol{l}_{\mathrm{f}}, \boldsymbol{p}_{\mathrm{i}}, \boldsymbol{l}_{\mathrm{i}}\right) \propto & \left|\tau_{\mathrm{f}} i\right|^{2} \int_{-\infty}^{\infty} \mathrm{d} t \mathrm{e}^{-\mathrm{i}\left(E_{\mathrm{f}}^{\mathrm{T}}-E_{\mathrm{i}}^{\mathrm{T}}+E_{\mathrm{f}}^{\mathrm{R}}-E_{\mathrm{f}}^{\mathrm{R}}\right) t / \hbar} \\
& \times K_{\mathrm{T}}\left(t, T_{S}\right) K_{\mathrm{R}}\left(t, T_{S}\right) .
\end{aligned}
$$

Starting from Eq. (6), the problem of determining the scattering probability now becomes one of choosing models for the scattering kernels for each of the energy exchange processes. For the exchange of phonons, an extension of the semiclassical model originally introduced by Brako and Newns for inelastic scattering of ions and atoms from smooth surfaces will be applied [6-8]. They showed the phonon scattering kernel can be expressed in the classical limit in terms of a general correlation function $Q_{\mathrm{T}}(\boldsymbol{R}, t)$ as

$$
K_{\mathrm{T}}\left(t, T_{S}\right)=\int_{-\infty}^{\infty} \mathrm{d} \boldsymbol{R} \mathrm{e}^{\mathrm{i} \boldsymbol{K} \cdot \boldsymbol{R}} \mathrm{e}^{-2 W_{\mathrm{T}}\left(\boldsymbol{p}_{\mathrm{f}}, \boldsymbol{p}_{\mathrm{i}}\right)} \mathrm{e}^{Q_{\mathrm{T}}(\boldsymbol{R}, t)},
$$

where $2 W_{\mathrm{T}}\left(\boldsymbol{p}_{\mathrm{f}}, \boldsymbol{p}_{\mathrm{i}}\right)=Q_{\mathrm{T}}(\boldsymbol{R}=0, t=0)$ is the contribution to the Debye-Waller factor due to phonons.

If the semiclassical limit is now extended to the limit of quick collisions in which the semiclassical force exerted on the scattering particle can be replaced by the momentum impulse, then the correlation function becomes the time dependent displacement correlation function

$Q_{\mathrm{T}}(\boldsymbol{R}, t)=\langle\boldsymbol{p} \cdot \boldsymbol{u}(0,0) \boldsymbol{p} \cdot \boldsymbol{u}(\boldsymbol{R}, t)\rangle / \hbar^{2}$,

where $\boldsymbol{u}(\boldsymbol{R}, t)$ is the phonon displacement vector at the position $\boldsymbol{R}$ on the surface.

The classical limit is obtained from Eq. (8) by making an expansion over small times and small position vectors around the point of collision which gives

$$
\begin{aligned}
\langle\boldsymbol{p} \cdot \boldsymbol{u}(0,0) \boldsymbol{p} \cdot \boldsymbol{u}(\boldsymbol{R}, t)\rangle / \hbar^{2} & \\
= & 2 W_{\mathrm{T}}\left(\boldsymbol{k}_{\mathrm{f}}, \boldsymbol{k}_{\mathrm{i}}\right)-\frac{i}{\hbar} t \Delta E_{0}-\frac{t^{2}}{\hbar^{2}} \Delta E_{0} k_{\mathrm{B}} T_{S} \\
& -\frac{\Delta E_{0} k_{\mathrm{B}} T_{S} R^{2}}{2 \hbar^{2} v_{\mathrm{R}}^{2}} .
\end{aligned}
$$

If the translational motion scattering kernel formed from Eq. (9) is used in the differential reflection coefficient of Eq. (6), the Debye-Waller factor is canceled by the leading term of Eq. (9). If in addition, rotation is ignored, i.e. if $K_{\mathrm{R}}\left(t, T_{S}\right)$ is set equal to unity, then the result for the differential reflection coefficient is just Eq. (4) above. This and similar theories have been successful in explaining the observed angular distributions and energy resolved spectra observed in several ion or atom scattering experiments [1,2].

The next task is to develop a scattering kernel for the rotational excitation of the molecular projectile. To be consistent with the smooth surface model of Eq. (9) for the translational motion, the model should preserve the correct angular momentum conservation for a rotating molecule interacting with a smooth surface, i.e. angular momentum will be conserved in the direction perpendicular to the surface but not in the directions parallel to the surface. Beginning with Eq. (5) in the semiclassical limit, but with the proper angular momentum conservation for a smooth 
surface, the scattering kernel for rotational excitation is

$K_{\mathrm{R}}\left(t, T_{S}\right)=\int_{-\infty}^{\infty} \mathrm{d} \theta_{z} \mathrm{e}^{\mathrm{i} l z \theta_{z} / \hbar} \mathrm{e}^{-2 W_{\mathrm{R}}\left(l_{\mathrm{f}}, \boldsymbol{l}_{\mathrm{i}}\right)} \mathrm{e}^{Q_{\mathrm{R}}\left(\theta_{z}, t\right)}$,

where $\boldsymbol{l}=\boldsymbol{l}_{\mathrm{f}}-\boldsymbol{l}_{\mathrm{i}}$ is the angular momentum transfer, $Q_{\mathrm{R}}\left(\theta_{z}, t\right)$ is a generalized rotational correlation function and the rotational Debye-Waller factor is $2 W_{\mathrm{R}}\left(\boldsymbol{l}_{\mathrm{f}}, \boldsymbol{l}_{\mathrm{i}}\right)=Q_{\mathrm{R}}\left(\theta_{z}=0, t=0\right)$. In the limit of a quick collision, where the angular forces are governed by the angular impulse, the correlation function becomes a function of the angular displacement $\Theta\left(\theta_{z}, t\right)$

$Q_{\mathrm{R}}\left(\theta_{z}, t\right)=\left\langle\boldsymbol{l} \cdot \boldsymbol{\Theta}(0,0) \boldsymbol{l} \cdot \boldsymbol{\Theta}\left(\theta_{z}, t\right)\right\rangle$.

The angular scattering kernel of Eqs. (10) and (11) is still fully quantum mechanical, although it is in the semiclassical limit. The extension to the classical limit of exchange of large numbers of rotational quanta is again similar to Eq. (9)

$$
\begin{aligned}
\langle\boldsymbol{l} \cdot & \left.\Theta(0,0) \boldsymbol{l} \cdot \Theta\left(\theta_{z}, t\right)\right\rangle \\
= & 2 W_{\mathrm{R}}\left(\boldsymbol{l}_{\mathrm{f}}, \boldsymbol{l}_{\mathrm{i}}\right)-\frac{i}{\hbar} t \Delta E_{0}^{\mathrm{R}}-\frac{t^{2}}{\hbar^{2}} \Delta E_{0}^{\mathrm{R}} k_{\mathrm{B}} T_{S} \\
& -\frac{\Delta E_{0}^{\mathrm{R}} k_{\mathrm{B}} T_{S} \theta_{z}^{2}}{2 \hbar^{2} \omega_{\mathrm{R}}^{2}}
\end{aligned}
$$

where

$\Delta E_{0}^{\mathrm{R}}=\frac{l_{x}^{2}}{2 I_{x x}^{c}}+\frac{l_{y}^{2}}{2 I_{y y}^{c}}+\frac{l_{z}^{2}}{2 I_{z z}^{c}}$

is the rotational recoil energy, the $I_{x x, y y, z z}^{c}$ are the principle moments of inertia of a surface molecule and $\omega_{\mathrm{R}}$ is a weighted average of frustrated rotational angular velocities of the surface molecules in the $z$-direction. The constant $\omega_{\mathrm{R}}$ plays a similar role for rotational transfers as the weighted average of parallel phonon velocities $v_{\mathrm{R}}$ in Eq. (4). Both of these quantities are fully determined if the complete dynamical structure function of the surface is known. For the purpose of this work, these quantities will be treated as parameters.

If the scattering kernels for both translational and rotational motion formed from Eqs. (9) and (12), respectively, are inserted back into Eq. (6) it again seen that all Debye-Waller factors disappear. This cancellation of the Debye-Waller factors is a characteristic of the classical limit. The final result for the differential reflection coefficient is then

$$
\begin{aligned}
& \frac{\mathrm{d} R\left(\boldsymbol{p}_{\mathrm{f}}, \boldsymbol{p}_{\mathrm{i}}\right)}{\mathrm{d} E_{\mathrm{f}}^{\mathrm{T}} \mathrm{d} \Omega_{\mathrm{f}}}=\frac{m^{2} p_{\mathrm{f}} L^{4}}{8 \pi^{2} \hbar^{2} p_{i z} S_{\mathrm{uc}}}\left|\tau_{f i}\right|^{2}\left(\frac{\pi v_{\mathrm{R}}^{2}}{\Delta E_{0} k_{\mathrm{B}} T_{S}}\right) \\
& \quad \times\left(\frac{2 \pi \hbar^{2} \omega_{\mathrm{R}}^{2}}{\Delta E_{0}^{\mathrm{R}} k_{\mathrm{B}} T_{S}}\right)^{1 / 2}\left(\frac{\pi}{\left(\Delta E_{0}+\Delta E_{0}^{\mathrm{R}}\right) k_{\mathrm{B}} T_{S}}\right)^{1 / 2} \\
& \quad \times \exp \left[-\frac{2 \boldsymbol{P}^{2} v_{\mathrm{R}}^{2}}{4 \Delta E_{0} k_{\mathrm{B}} T_{S}}\right] \exp \left[-\frac{2 l_{z}^{2} \omega_{\mathrm{R}}^{2}}{4 \Delta E_{0} k_{\mathrm{B}} T_{S}}\right] \\
& \quad \times \exp \left[-\frac{\left(E_{\mathrm{f}}^{\mathrm{T}}-E_{\mathrm{i}}^{\mathrm{T}}+E_{\mathrm{f}}^{\mathrm{R}}-E_{\mathrm{i}}^{\mathrm{R}}+\Delta E_{0}+\Delta E_{0}^{\mathrm{R}}\right)^{2}}{4\left(\Delta E_{0}+\Delta E_{0}^{\mathrm{R}}\right) k_{\mathrm{B}} T_{S}}\right] .
\end{aligned}
$$

Eq. (13) is the fundamental result of this work and forms the basis for the calculations shown below. Its main features consist of a Gaussian-like function in the translational and rotational energy exchange together with the recoil energies, with a width that increases as the square root of the product of temperature $T_{S}$ and the sum of the recoil energies. It is multiplied by a product of envelope functions that depend inversely on the recoil energies and on $T_{S}^{2}$. There are also Gaussianlike functions in the momentum transfer $\boldsymbol{P}$ and the angular momentum transfer $l_{z}$ which arise as a result of the correct momentum conservation for a smooth surface. These Gaussian-like functions are not true Gaussians, however, because of the momentum dependence of the recoil energies.

All quantities that can be measured can be calculated from the state-to-state differential reflection coefficient Eq. (13). As an example, the total angular distribution of molecules scattered from a well defined incident beam, regardless of final translational energy or rotational energy, is obtained by summing over initial and final angular momenta and translational energy,

$\frac{\mathrm{d}^{2} R}{\mathrm{~d} \Omega f}=\int_{0}^{\infty} \mathrm{d} E_{\mathrm{f}}^{\mathrm{T}} \int \mathrm{d} \boldsymbol{l}_{\mathrm{f}} \int \mathrm{d} \boldsymbol{l}_{\mathrm{i}} \mathscr{P}\left(\boldsymbol{l}_{\mathrm{i}}\right) \frac{\mathrm{d} R}{\mathrm{~d} \Omega_{\mathrm{f}} \mathrm{d} E_{\mathrm{f}}^{\mathrm{T}}}$,

where $\mathscr{P}\left(\boldsymbol{l}_{\mathrm{i}}\right)$ is the distribution function for initial angular momenta in the incident beam, which in many experiments is approximated by a MaxwellBoltzmann distribution with a small rotational temperature. Other quantities, such as the average energies, can be calculated by using Eq. (13) as the distribution function for scattered molecules. 
As stated above in the introduction, the initial motivation for this work was the innovative experiment of Miller et al. for the scattering of $\mathrm{C}_{2} \mathrm{H}_{2}$ from $\mathrm{LiF}\left(\begin{array}{ll}0 & 0\end{array}\right)$. For fixed angle incident molecular beams and over a large range of incident translational energies they made three different sets of experimental measurements: (1) total angular distributions, i.e. intensities as a function of final angle, (2) scattered intensities as functions of final rotational energy measured at fixed final angle and (3) final rotational temperatures of the scattered beam at fixed final angle.

All of these quantities were calculated earlier with a simpler molecular scattering model that did not include the correct conservation of angular momentum at a smooth surface [4]. This earlier model did a satisfactory job of describing the measured angular distributions, but gave only qualitative agreement with the data for the measurements of the final rotational excitations.

Calculations of the angular distributions with the present model, using Eq. (14) are in equally good agreement with the experimental data as were the earlier calculations, thus they are not presented here. The earlier conclusions based on the agreement with the angular distribution calculations, was that the mechanism determining the shape of the angular distribution lobes was dominated by multiphonon energy transfer to the surface. The present calculations sustain that conclusion.

Fig. 1 is an example of the experimental data, shown as circles, for the scattered intensity measured as a function of final rotational energy $E_{\mathrm{f}}^{\mathrm{R}}$ compared with calculations of the present theoretical model shown in the solid curve. The incident translational energy is $E_{\mathrm{i}}^{\mathrm{T}}=242 \mathrm{meV}$, the incident angle is $\theta_{\mathrm{i}}=60^{\circ}$ and the final angle is at the maximum of the angular distribution $\theta_{\mathrm{i}}=47^{\circ}$.

There are two parameters involved in the calculations of Fig. 1, the weighted averages of surface-parallel phonon speeds $v_{\mathrm{R}}$ and the weighted average of frustrated rotational frequencies $\omega_{R}$ of the $\mathrm{LiF}$ molecules in the $z$-direction. In principle, these are quantities that can be calculated but in this work we have used them as parameters. The value of $v_{\mathrm{R}}$ is expected to be less than the Rayleigh phonon velocity [6] and for the case of the calculations shown in Fig. 1 this is chosen to have the

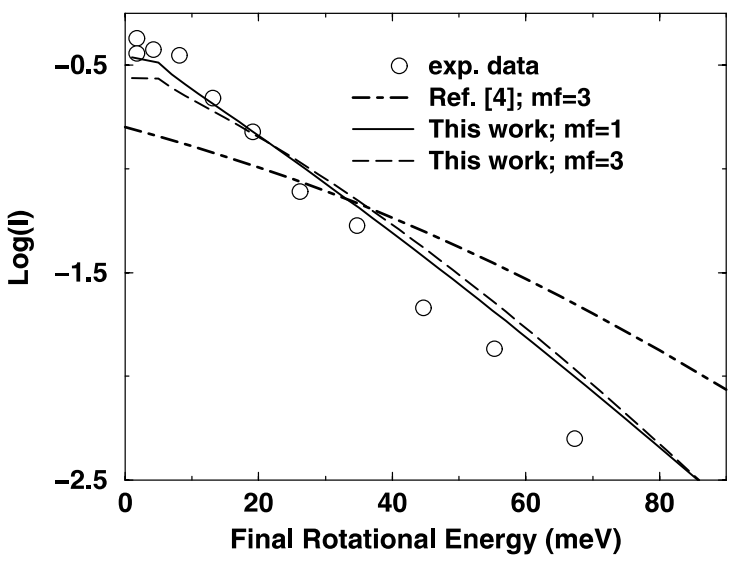

Fig. 1. The scattered intensity as a function of final rotational energy $E_{\mathrm{f}}^{\mathrm{R}}$. The incident translational energy is $242 \mathrm{meV}$, the incident angle is $\theta_{\mathrm{i}}=60^{\circ}$ and the final angle $\theta_{\mathrm{f}}=47^{\circ}$ is at the maximum in the corresponding measured angular distribution. The experimental data from [5] are shown as points and the present calculations are the solid curve. The dash-dotted curve shows calculations from an earlier model with an effective surface mass three times that of the LiF molecule $(m f=3)$ [4] and the dashed curve is the present calculation with $m f=3$.

value $450 \mathrm{~m} / \mathrm{s}$, consistent with the choice that made for the best agreement with the measured angular distributions at that same incident translational energy. In order to get a best fit with the measured angular distributions, the parameter $v_{\mathrm{R}}$ ranged from $200 \mathrm{~m} / \mathrm{s}$ at the lowest measured translational energy of $94 \mathrm{meV}$ to $1200 \mathrm{~m} / \mathrm{s}$ at the highest energy of $618 \mathrm{meV}$. The value of $\omega_{\mathrm{R}}$ was chosen to be $10^{13} \mathrm{~s}^{-1}$, but the results are insensitive to this choice within an order of magnitude. All other parameters such as the surface molecular mass and the moments of inertia of $\mathrm{C}_{2} \mathrm{H}_{2}$ and $\mathrm{LiF}$ were calculated using known atomic masses and molecular bond lengths.

Also shown in Fig. 1 as a dash-dotted curve is the calculation of the earlier theoretical model [4], which were obtained using an effective mass for the surface which is three times the mass of a $\mathrm{LiF}$ molecule. For comparison, the present calculations, using an effective mass of three LiF molecules is shown in the dashed curve. Clearly, the present calculations with the proper LiF mass give reasonable agreement with experiment, while it is seen that the more restrictive earlier model agrees only qualitatively. 
In the semi-logarithmic plot of Fig. 1 the experimental data points lie to a reasonable approximation along a straight line, indicating exponential dependence of the intensity on $E_{\mathrm{f}}^{\mathrm{R}}$. If this exponential dependence is compared to a Maxwell-Boltzmann distribution, a final rotational temperature can be extracted at each incident translational energy $E_{\mathrm{i}}^{\mathrm{T}}$ and this procedure was used in the original interpretation of the data [5]. Fig. 2 shows the experimental values of the final rotational temperatures $T_{\mathrm{fR}}$ plotted as a function of incident translational energy and compared to the present calculations exhibited as the solid curve. The calculated final rotational temperatures were extracted from curves such as shown in In Fig. 1 by least-squares fitting to a straight line at energies $E_{\mathrm{f}}^{\mathrm{R}}>8 \mathrm{meV}$, where the calculations reasonably approximate straight lines. Also shown in

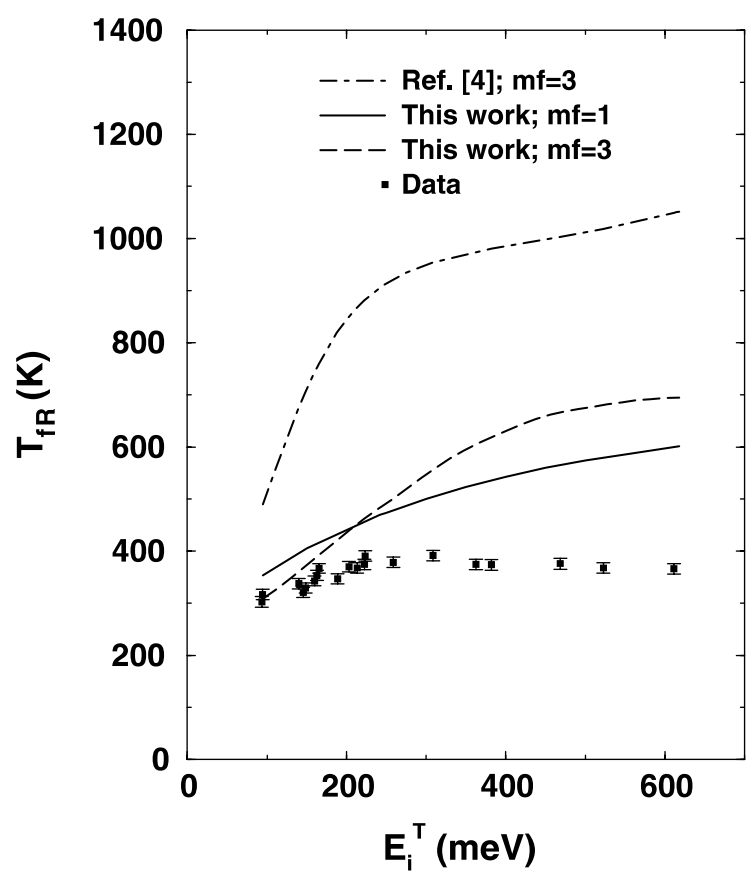

Fig. 2. Final rotational temperatures $T_{\mathrm{fR}}$ plotted as a function of incident translational energy $E_{\mathrm{i}}^{\mathrm{T}}$. The incident angle is $\theta_{\mathrm{i}}=60^{\circ}$ and the final angle is at the maximum in the corresponding angular distribution for the incident energy. The experimental data from [5] are shown as points with error bars, the present calculations are the solid curve, the results of earlier calculations [4] are shown in the dash-dotted curve and the present calculations with $m f=3$ are shown in the dashed curve.
Fig. 2 as a dash-dotted line are the results of the earlier calculation [4] with an effective mass of three LiF molecules. For comparison, the dashed curve shows calculations with the present model with the same larger effective mass. The earlier calculations are in only qualitative agreement with experiment and greatly overestimate the final rotational temperatures. The present calculations, while much better than the earlier results, are still significantly larger than the experimentally measured values. Thus an appropriate conclusion to draw from this work is that a scattering theory that accounts for the correct momentum conservation laws at a smooth surface gives better agreement with the measurements, the description still cannot be termed satisfactory. More recent calculations, for a non-rigid molecule that includes the possibility of excitation of internal molecular modes of vibration, agree nearly quantitatively with the measurements and shows that internal mode excitation is also very important in the scattering process [10].

In this paper a classical theory of translational and rotational motion has been used to treat the scattering of a molecular beam by a surface. This is an extension of earlier work [4] to include the correct conservation of both linear and angular momentum in the collision of the molecule with a smoothly varying surface. The results of calculations of this theoretical model are compared with recent experimental measurements for the scattering of $\mathrm{C}_{2} \mathrm{H}_{2}$ from $\mathrm{LiF}\left(\begin{array}{lll}0 & 0 & 1\end{array}\right)$ [5]. Quite good agreement is obtained with the measured scattered angular distributions, just as was the case with the previous theoretical model. This good agreement, together with further calculations that demonstrate that the calculated angular distributions do not depend strongly on the rotational excitations, are taken as evidence that the shape of the measured angular distribution lobes is most strongly dictated by exchange of large numbers of phonons. Further comparisons with experiment are made for the scattered intensities as functions of final rotational energy and for the final rotational temperatures as functions of the incident beam translational energy. In both of these cases, the theoretical calculations are considerably improved over the results of the previous simpler scattering model [4]. 
This work shows that for molecules as heavy as $\mathrm{C}_{2} \mathrm{H}_{2}$, with translational energies as large as $100 \mathrm{meV}$, classical physics is sufficient to describe rotational and phonon excitation. It also shows that correct application of the conservation laws for angular momentum exchange and linear momentum exchange at a smoothly varying surface are of vital importance in describing the scattering process. Another important point of the present work is that this theoretical approach results in a closed-form equation for the scattering intensities. Having such a closed-form expression is useful for readily determining how the scattered intensity will respond to changes in the incident parameters, such as incident energies, incident angles and surface temperatures.

\section{Acknowledgements}

The authors would like to thank I. Iftimia and H. Zhang for valuable discussions during the course of this work. This work was supported by the National Science Foundation under grant number DMR-0089503 and by the Department of Energy under grant number DEFG0298ER45704.

\section{References}

[1] A. Muis, J.R. Manson, Phys. Rev. B 54 (1996) 2205.

[2] A. Muis, J.R. Manson, J. Chem. Phys. 111 (1999) 730.

[3] M.F. Bertino, J.R. Manson, W. Silvestri, J. Chem. Phys. 108 (1998) 10239.

[4] H. Zhang, J.R. Manson, J. Chem. Phys. 113 (2000) 8290.

[5] T.W. Francisco, N. Camilone, R.E. Miller, Phys. Rev. Lett. 77 (1996) 1402.

[6] R. Brako, D.M. Newns, Phys. Rev. Lett 48 (1982) 1859; R. Brako, D.M. Newns, Surf. Sci. 123 (1982) 439.

[7] H.-D. Meyer, R.D. Levine, Chem. Phys. 85 (1984) 189.

[8] V. Bortolani, A.C. Levi, Rivista del Nuovo Cimento 9 (1986) 1.

[9] J.R. Manson, V. Celli, D. Himes, Phys. Rev. B 49 (1994) 2782.

[10] I. Iftimia, J.R. Manson, Phys. Rev. Lett. 87 (2001) 093201. 